\title{
Algunas problemáticas y tensiones en el campo educativo y la formación
}

\section{Problems and tensions in the educational and formation field}

DOI: https://doi.org/10.32870/dse.v0i12.264

José Luis Martínez Rosas*

Los inicios de la conformación del campo educativo en tanto campo de conocimiento podemos situarlos de manera arbitraria en la obra pionera de Juan Amos Comenio en el siglo XVI, desde entonces este campo ha sido y continúa siendo un espacio sujeto a tensiones y problemáticas.

Algunos de sus elementos son y deben ser sometidos a un amplio debate a la luz de las condiciones y necesidades contemporáneas. En una enumeración nada exhaustiva se han sometido a análisis críticos y debates elementos tales como:

1. Los tipos de escuela: moderna, tradicional o activa e incluso la propia desescolarización; y el carácter de sus sujetos: pasivos o activos.

2. Las relaciones entre "lo educativo", "la educación" y "la formación".

3. Los fines y sentido de la educación y la formación.

4. La primacía de los aspectos cognitivos, sociales o morales en los procesos de formación.

5. La denominación del campo: educación, pedagogía, ciencia educativa o ciencias de la educación.

6. El estatus epistemológico de la disciplina: ¿se trata efectivamente de una disciplina o de un campo de prácticas sociales, de un conjunto de saberes profesionales o de una ciencia?

7. La existencia de un objeto teórico propio del campo y de algún método de investigación específico para su estudio, así como las relaciones entre los discursos teóricos y metateóricos o epistémicos.

8. El margen de autonomía relativa de este campo frente a otros, así como las relaciones intercampos.

* Doctor en Ciencias de la Educación. Docente en diversas universidades. integrante de la Red de Posgrados en Educación y de la Red de Investigación sobre la Formación y Asesoría de Posgrado en México. Correo electrónico: joseluismar444@hotmail. com 
9. El grado de permeabilidad de este espacio social y las características de su estructuración interna, así como las normas, exigencias y el tipo de capital necesarios para el ejercicio de las profesiones propias del campo, entre ellas la docencia.

10. Las relaciones entre los proyectos educativos y los proyectos de nación, de Estados-nación o de Estados-posnacionales; así como la contribución de la educación al desarrollo económico y social o a la disminución de la desigualdad socioeconómica.

11. Las características y relaciones entre la teoría y las prácticas docentes, de gestión, de investigación, etcétera.

12. Las relaciones hegemónicas o de subordinación entre las distintas prácticas, por ejemplo, el papel de la docencia y el de la investigación educativas; o la función de la enseñanza ante los conocimientos surgidos en las ciencias duras o en las humanidades: trasmisión, interpretación, transformación de los objetos científicos en objetos de enseñanza, etcétera.

13. Las tensiones y nexos entre educación y cultura y entre homogeneidad y diversidad cultural.

14. Las relaciones entre actores sociales, económicos, políticos y educativos, tanto nacionales como globales, y la naturaleza de las reformas educativas.

Los asuntos anteriores son apenas un botón de muestra que nos permite percibir los múltiples e importantísimos asuntos en debate, lo cual resulta necesario para visualizar la complejidad del campo y dimensionar la gran diversidad de aspectos susceptibles de crítica y debate.

En este orden de ideas, precisamos que se trata a fin de cuentas de facilitar la interlocución entre autor y lector para producir sucesivas interpelaciones y ampliar el marco de intersubjetividad con el propósito último de posibilitar una futura acción simbólica con sentido estratégico entre un número creciente de asociados.

Por lo anterior, le proponemos al lector centrarnos en la homogeneización de dos componentes del espacio educativo que inciden en los procesos de formación: la escuela y el currículum, a fin someter el proceso de homogeneización a debate:

\section{Las tensiones y exigencias en el espacio escolar}

La educación escolarizada en todos los niveles se constituye por distintos elementos, entre ellos: a) la institución escolar con su organización, su infraestructura, equipamiento y otros constitutivos de mayor complejidad, b) el currículum, c) la didáctica, d) las prácticas educativas y los recursos pedagógicos, e) los sujetos del acto educativo y f) otros sujetos sociales.

En muchas ocasiones se afirma en círculos de docentes que la educación es la misma desde hace siglos, sin embargo, en contra de esta idea la educación escolarizada en sus diferentes niveles ha sufrido cambios notorios desde el surgimiento del capitalismo y la modernidad; y en la actua- 
lidad la naturaleza del espacio escolar: cultural, política, científica, socializadora, moralizante, ha sido "puesta en cuestión", criticada desde posicionamientos que suelen denominarse como "neoliberales", a fin de que la institución escolar se integre y sea plenamente funcional a las agendas educativas globales y los proyectos educativos de los Estados-nación en tránsito hacia Estadosposnacionales. Debido a esta crítica, el conjunto de la educación formal y la escuela se encuentran en una situación de exigencia sufriendo múltiples tensiones y acomodos que no satisfacen ni a los círculos dirigentes de los sistemas educativos nacionales, ni a los organismos directores de los procesos metanacionales, ni a otros actores como es el caso de los dirigentes empresariales.

En las últimas décadas se han llevado a cabo una diversidad de procesos de reforma de la educación escolarizada cuyo propósito ha consistido en ponerla a tono con las exigencias del entorno, ya sea reorganizando el sistema, ya dirigiéndola hacia una mayor calidad. Al iniciar la segunda década del siglo XXI tales cambios han derivado en una progresiva homogeneización tanto del modelo de institución escolar como del currículum, y paralelamente en una estandarización de la evaluación, de tal manera que pueden reconocerse los rasgos similares de estas tres entidades en distintos lugares del orbe.

La homogeneización, que en este caso se aplica a la institución escolar y al currículum, proviene del término latino homogenéus y refiere o predica del sujeto o entidad que pertenece a un mismo género o conjunto, posee elementos o características iguales o uniformes (RAE, 2014); sin embargo, lo homogéneo no implica olvidar que a la par de lo igual y uniforme existen elementos de diferenciación que aportan rasgos diversos a las escuelas o a los currícula.

La crítica hacia las limitaciones de la educación hecha desde organismos como la Organización para la Cooperación y el Desarrollo Económico (OCDE) es un medio para lograr la subordinación y subsunción de la institución escolar y el currículum a la agenda neoliberal y homogeneizar sus características básicas.

Develar lo anterior es necesario para debatir y criticar este proyecto de instalar un solo modelo de escuela y un solo tipo de currículum, promoviendo una diversidad de proyectos educativos, de escuela y de currículum alternativos acordes a los diferentes proyectos de cultura, sociedad y economía que pudieran existir.

\section{La homogeneización del modelo de institución escolar}

A partir del cierre de la edad media, que generalmente se da por finalizada con la caída del Imperio Bizantino en 1453 o por el descubrimiento de América en 1492, y de la configuración del propio sistema-mundo capitalista y moderno, se pueden distinguir dos grandes períodos en el desarrollo histórico de la institución escolar: el de la escuela tradicional y el de la escuela moderna.

La escuela tradicional, de acuerdo con la indagación de Palacios (2007), se constituyó en Europa hacia el siglo XVII con los colegios-internados y, en buena medida, a partir del éxito de los conducidos por los jesuitas. Los rasgos principales de este modelo de escuela, que son el método 
y el orden a decir de Jesús Palacios, son sistematizados por Comenio en su didáctica magna publicada en 1657.

Se desarrollaron al menos dos variantes de la escuela tradicional, la originaria de los colegiosinternados y la de la escuela tradicional reformada.

En general la vida cotidiana de los colegios-internados era una lección de moral. Los colegiosinternados se caracterizaron por su separación del mundo y por la vigilancia constante sobre el alumno (Snayders, cit. en Palacios, 2007), el contenido de su enseñanza es el retorno a la antigüedad, la lengua de uso cotidiano es el latín, la enseñanza-aprendizaje se basaba en el arte de la retórica (Mesnard, 1974) y en un sistema de competencia entre griegos y cartagineses con émulos en cada bando; la clase o grupo se ordenaba jerárquicamente en magistrados, decuriones y otras categorías, y el maestro organizaba la vida y actividades del internado al mismo tiempo que vigilaba el cumplimiento de las reglas establecidas.

Las reformas a la escuela tradicional que impulsaron Comenio y otros en Europa incluían un cambio en la lengua escolar del latín por la lengua nativa como el moravo, la incorporación de conocimientos útiles y la escolarización universal a cargo del Estado para hombres y mujeres, pobres y ricos.

Mientras que la escuela moderna recoge el legado de la modernidad, opuesta a la edad antigua y la época medieval que dominó el escenario europeo hasta el siglo XV y la primera mitad del XVI, la escuela "moderna" típica correspondía a la era de la revolución industrial y las expectativas sobre ella eran amplias y firmes, se tenía la convicción de que era factible una formación general cercana a las humanidades y que la escuela media tenía una función propedéutica en la enseñanza de las ciencias y la preparación para el trabajo (González, cit. en Palacios, 2007).

El ideario de la modernidad incluye una esfera pública vertebrada por la noción de ciudadanía y el Estado-nación como garante de los derechos y deberes de los individuos, el impulso a la producción capitalista, a la mercantilización y a la conquista y constitución de colonias, el desarrollo de pensamiento y la racionalidad ligados al despliegue de las ciencias y disciplinas, el cuestionamiento del sistema hermenéutico imperante en materia religiosa y el surgimiento del protestantismo y la reforma religiosa, entre otros elementos a los que se asocian hechos como la invención de la imprenta y el descubrimiento de América.

Recurriendo en buena medida a Michel Foucault (1986), la escuela moderna es un dispositivo de poder, saber y formación requerido para incorporar en los educandos la disciplina necesaria para el desarrollo del pensamiento racional y lógico propio del capitalismo.

La escuela moderna funciona como dispositivo, el cual de acuerdo con Martha Souto se entiende desde la perspectiva foucaultiana como "una red que vincula un conjunto de elementos heterogéneos en un juego de las relaciones de poder y de saber con un carácter estratégico" (Souto, 1999: 70); y como tal entiende la vigilancia, el control, la disciplina e incluso el castigo como elementos que posibilitan su carácter estratégico vinculado además al deseo y esperanza de emancipación social. 
Consideramos que la escuela moderna no sólo es dispositivo, sino que su carácter estrictamente simbólico es también una institución que sedimenta normas y valores, en este caso, que realza los códigos culturales de la alta cultura, el saber y la transmisión escrita a través de los libros que la imprenta hace factible producir en serie, al grado de que maestro y libro representan la autoridad, el saber y el poder; en síntesis, es la escuela de la racionalidad y el pensamiento ilustrado exigidos por el despliegue de las ciencias naturales y sociales.

Las diversas variantes de la escuela nueva, cuyas raíces se remontan hasta Rousseau (2005), expresan los distintos énfasis que uno o más de los rasgos mencionados adquieren en la propuesta de cada pedagogo; Cousinet se centra en el trabajo de equipo, Freinet en la exploración del medio y el uso de la imprenta y el texto, Claparede y Dewey buscan cada uno a su manera impulsar una educación funcional, Dewey enfatiza el carácter democrático de la educación incluso renovando la escuela como taller o laboratorio, el propio Makarenko, a quien muchos excluyen de las epopeyas pedagógicas de la escuela nueva, trabaja en la disciplina, la emulación y el inculcamiento de valores colectivos, entre otros.

De todas estas propuestas emergerá poco a poco un modelo unificado de institución escolar que compartirán con sus diferenciaciones particulares las naciones y hasta las facciones en lucha en las distintas guerras de independencia en las otrora colonias y posteriormente en las guerras civiles; visto a lo lejos, es un proceso de unidad con diversidad donde la institución escolar moderna que hoy conocemos se despliega históricamente.

Sin duda la concepción contemporánea de un modelo más o menos homogéneo de escuela para cada uno de sus tres ciclos de educación (básica, media y superior), al parecer más homogéneo en el segmento de la educación básica y más diverso en el de educación superior, sigue impregnada de aquellas características. Incluso puede afirmarse que la educación básica extendida (EBE) que se proyecta hasta una parte de la educación media, al incluir competencias genéricas, refina y precisa lo que hoy se espera en todo el planeta respecto a un individuo que posee los elementos mínimos suficientes para actuar en la sociedad contemporánea globalizada.

La EBE es deudora de estas exigencias y expectativas sobre el proyecto de escuela moderna, pero también muestra sus limitaciones.

Respecto a la educación media y de acuerdo con Santos del Real (2000), los jóvenes tienen claro que el certificado de secundaria y los estudios más allá de este nivel son condición necesaria pero insuficiente para acceder o mejorar su condición ocupacional; además, que los jóvenes en edad de educación secundaria asocian al desempeño docente su gusto por el aprendizaje y su percepción sobre la relevancia de los contenidos escolares. Este conjunto de situaciones se suma a las exigencias que el contexto globalizado marca a este nivel educativo, por lo que se exige no sólo un cambio a estas escuelas, sino a sus docentes.

Sirva esto como muestra de que la escuela moderna ha sido erosionada en la actualidad perdiendo eficacia en la constitución de la subjetivad requerida por las condiciones del actual contex- 
to; pero, al parecer, el espacio vacío generado por la pérdida de eficacia se cubre con una mayor competencia y competitividad exigida desde otro rasgo del modelo homogeneizado: la mercantilización progresiva de la escuela.

Las escuelas mercantilizadas son un signo de nuestra época. El Banco Mundial establece entre sus propuestas más prototípicas la autonomía escolar, que en buena medida se ha interpretado no sólo como autonomía de gestión, sino como desprendimiento de la escuela de su carácter estadual, para asumir un nuevo contrato a manera de empresa privada con sus clientes, sean alumnos y/o padres de familia.

El ideario de la modernidad unas veces combatido y otras subsumido en el pensamiento neoliberal ha corrido igual suerte que la escuela moderna, la cual siendo dispositivo de formación de sujetos acordes a la modernidad, ahora por medio de su homogeneización, se convierte en un dispositivo altamente eficaz para instalar en las mentes, los cuerpos y los corazones de los sujetos los rasgos performativos que el pensamiento neoliberal exige.

¿Este dispositivo escolar homogeneizado y homogeneizante es acaso el necesario para preservar y desarrollar la gran diversidad de opciones culturales, económicas, políticas y sociales de la humanidad? ¡Evidentemente no!

\section{La homogeneización del currículum}

Así como hemos tratado de mirar la educación, la formación y la escuela más allá de sí mismas, es menester hacer lo propio con el currículum. Sin duda las reformas curriculares, consideradas como reformas de tercera generación que acompañan a las reformas centradas en la planeación, evaluación y gestión educativas, dan cuenta de un tramo en la historia cultural y no sólo de la historia escolar, la historia cultural es un término propio

del campo histórico más amplio que da la atención a los giros lingüísticos en los estudios sociales e históricos (Popkewitz, Franklin y Pereyra, 2003). La preocupación última está con los sistemas de la razón que ordena y clasifica los objetos de escolarización (las nociones de niñez, materias escolares, teorías de enseñanza y aprendizaje). El énfasis en el currículum es hacer del problema del conocimiento y del razonamiento en las escuelas, las formas por las cuales nosotros "contamos la verdad" sobre nosotros mismos y otros, como lugar central para entender la formación de la escolarización moderna.

En este orden de ideas, los cambios en el espacio de la teoría curricular y en las mismas reformas curriculares pueden ser considerados como cambios en la historia cultural. Las categorías estelares que han vertebrado las propuestas curriculares asociadas a la escuela moderna son tres: formación, aprendizaje y competencia, y particularmente esta última se asocia al pensamiento neoliberal y al despliegue de la escuela como dispositivo performativo. 
En el contexto del período de proyectos de la cultura moderna y del capitalismo imperialista de la post-guerra fría, donde la dialéctica centros-periferias de la cultura-mundo y de la economíamundo pareciera ser la tensión más visible, aparece la categoría "competencia" con un papel estelar. De acuerdo con De Ketele (2008), se trata de un "concepto mal definido, poco claro y epistemológicamente dudoso", pero que ha llevado a que actualmente "el conocimiento se visualiza no como posesión de información sino competencia para resolver problemas ambiguos y cambiantes" (Rodríguez, cit. en Navarro Leal, 2010). Ante esta exigencia la escuela como dispositivo es a la vez más necesaria y más insuficiente (Filmus y otros, 2001), por lo que se encuentra ante mayores retos.

Vinculadas a la globalización neoliberal y a la economía del conocimiento, las reformas educativas de primera y segunda generación que impulsan las políticas de calidad: la planeación, rendición de cuentas, evaluación, certificación y acreditación, se ven acompañadas a nivel mundial por el enfoque de competencias (Díaz-Barriga, 2011). Es tal la relevancia de esta categoría, que se constituye en la columna vertebral de las reformas curriculares de manera progresiva y se articula a un discurso más genérico sobre las características de la educación en tiempos de la globalización, por lo que se convierte en una narrativa que configura "lo educativo" y trasforma el sentido original de la formación integral sustituyéndolo por lo que Lyotard denomina "performatividad".

Las categorías de aprendizaje y formación sufren diversos efectos cuando la categoría "competencia", proveniente de los campos de la economía y administración, toma una posición estelar y articuladora en los discursos de política educativa y en los saberes de académicos y docentes en los finales del siglo XX, hasta obtener un papel central en la construcción del currículum y, finalmente, provocar el estallido, desplazamiento o anulación de la categoría "formación integral" y la subsunción de la categoría "aprendizaje" (Martínez, López y Carpio, 2013).

El surgimiento de la educación basada en normas de competencia en los EU hacia 1930 es el antecedente más remoto relativo a la génesis de la categoría "competencia", la cual surge en el ámbito económico (Arguelles, 1996; Gonczi, 1996); al respecto, según el Consejo de Normalización y Certificación de Competencias Laborales de México (SEP / CONOCER, 2008):

[Una] competencia laboral es la capacidad productiva de un individuo, que se define y se mide en términos de desempeño en un determinado contexto laboral, y no solamente como conocimientos, habilidades, destrezas y actitudes; éstas son necesarias, pero no suficientes por sí mismas para un desempeño efectivo.

Un segundo momento en el desarrollo de esta categoría como eje vertebrador del currículum se encuentra en la suscripción del Pacto de Bolonia y la creación del Espacio Europeo de Educación Superior por los ministros de educación de veintiún países de la Unión Europea en 1999; dicho pacto se orienta hacia la homologación de carreras y títulos mediante el sistema de créditos y la estandarización de cartas descriptivas en el currículum de las carreras; todo con el objeto de facilitar el libre tránsito de profesionistas y de estudiantes entre los países de la Unión. 
Casi inmediatamente, en 2000 y 2001, la Organización para la Cooperación y el Desarrollo Económico (OCDE) difunde la Definición y Selección de Competencias (DeSeCo), un documento clave para la diseminación de esta categoría en los sistemas educativos nacionales de sus países miembros, la cual se instala y sedimenta a través de las normas y programas educativos nacionales y sobre todo mediante el diseño y la aplicación periódica de la prueba PISA para evaluar las competencias de los jóvenes de 15 años y comparar cómo los sistemas nacionales y las escuelas funcionan eficazmente como dispositivos de performatividad cumpliendo cabalmente su cometido en la agenda educativa neoliberal.

El desarrollo del Proyecto Tunning para Europa en 2001 y para América Latina en 2004 marca la siguiente etapa en el despliegue de la categoría competencia, siendo uno de sus objetivos el diseño curricular para estudios superiores en diferentes profesiones basados en este enfoque. México es miembro de este último proyecto, lo cual ha llevado a que desde 2012 el enfoque por competencias se convierta en el eje de construcción curricular en todos los niveles de educación.

Como puede apreciarse, inicialmente la capacitación en el ámbito laboral con base en normas de competencia, cuyo sentido consistía en complementar lo que los trabajadores adquirían en las instituciones educativas, ha desembocado en un enfoque que vertebra los diseños curriculares en los diversos niveles educativos a lo largo no sólo de los Estados Unidos de Norteamérica, sino de la unión Europea y América Latina la educación, reconociéndose diversos momentos en el proceso de su instalación y sedimentación como categoría "estelar" en el espacio curricular.

Se ha desplazado la categoría formación, que se refiere al proceso de adquisición de cultura del espíritu, capacidad de juicio ético y estético, sabiduría y ciencia, sentido común y razón, saberes prácticos y técnicos, ideal del sabio y el erudito y que sintetiza el significado de "humanidad" (Gadamer, 1991-92); pero existe una cierta continuidad con el énfasis que la formación ha tenido en el mundo anglosajón entendiéndose como profesionalización. Este énfasis se ha heredado, como puede apreciarse en dos definiciones estelares acerca de lo que significa una competencia, para la Unión Europea (UE) y para la OCDE.

En el documento Educación y formación, la Unión Europea (2010) define una competencia como un "paquete multifuncional y transferible de conocimientos, destrezas y actitudes que todos los individuos necesitan para su realización y desarrollo personal, inclusión y empleo".

Mientras que en el Informe DeSeCo, la OCDE (2001) establece que una competencia es:

[La] capacidad de responder a demandas complejas y llevar a cabo tareas diversas de forma adecuada. Supone una combinación de habilidades prácticas, conocimientos, motivación, valores éticos, actitudes, emociones y otros componentes sociales y de comportamiento que se movilizan conjuntamente para lograr una acción eficaz. 
En los dos casos, se trata de un grupo de capacidades y acciones que se ponen en juego en situaciones de la vida no necesariamente escolar, con lo que adquieren su carácter performativo, en los que se demuestra que la persona se "realiza" o se "desempeña" adecuadamente de acuerdo con las exigencias que le plantean las circunstancias.

Es justamente en la exigencia de un desempeño eficaz donde existe mayor distancia con la adquisición de la cultura del espíritu, ya que una formación profunda y sólida en ámbitos del conocimiento que no necesariamente sean importantes en un "desempeño", tienden a ser omitidos en los procesos educativos basados en competencias. Ya Gimeno Sacristán (1990) había analizado cómo la "pedagogía por objetivos", muy similar a esta nueva "pedagogía del desempeño", estaba obsesionada por la eficiencia en los resultados educativos; es decir, por la visibilidad de los aprendizajes, operando desde una racionalidad "técnica" más preocupada por el uso de recursos que por los procesos de formación.

La anulación de la categoría "formación" es el signo de un énfasis en la instrumentalización de la educación y eventualmente de su total subordinación a las exigencias del mundo económico, a través de la competitividad y su sistema de valores (De la Torre, 2004), muy ligados al pragmatismo filosófico y al neoliberalismo económico. Una manera en la que se expresa esta condición es la pérdida de importancia de la formación en filosofía y epistemología en los posgrados en educación en México en la década 2002-2012 (Pérez, Limón y García, 2013).

A pesar de la débil consistencia teórica de los enfoques por competencias (Moreno Olivos, 2009), las reformas educativas de última generación en México y el mundo se han realizado desde este posicionamiento (Días Barriga, 2011); así se manifiesta en diversos estudios, por ejemplo: Lugo y colaboradores (2008) documentan y analizan cómo en la educación superior mexicana se ha transitado de las reformas educativas hacia las innovaciones curriculares y la intervención en las prácticas educativas a partir del enfoque por competencias; mientras que Barrón (2009) presenta el desarrollo de currículos por competencias como proyectos educativos innovadores en la educación superior mexicana; también la Secretaría de Educación Pública (SEP, 2008 y 2011) ha realizado diversas reformas de los planes de estudio en educación básica y media superior bajo el mismo enfoque en años recientes; asimismo, en los posgrados en educación la formación en filosofía y teoría educativa ha perdido relevancia frente a la formación en competencias (Pérez Arenas, 2007).

\section{Palabras finales}

En los distintos momentos y geografías que hemos recorrido brevemente se busca manifestar que la tendencia presente en México, Europa o los Estados Unidos es un camino hacia la homogeneidad curricular, ligada a una racionalidad técnica.

La escuela asociada al proyecto emergente del capitalismo y la modernidad posteriores a la edad media se ha trastocado buscando convertirla en un eficaz dispositivo performador asociado al pensamiento neoliberal. 
Los diseños curriculares y las instituciones educativas sufren continuos acomodos y reformas que son parte de un proceso general de homogeneización cuyo sentido, sin duda, trata de encontrar vías alternas de educación y de formación y, al mismo tiempo, poner un dique a la diversidad de opciones culturales y sociales divergentes a la así llamada globalización neoliberal.

\section{Referencias}

Arguelles, A. (comp.) (1996). Competencia laboral y educación basada en normas de competencia. México: CONALEP.

Barrón, Concepción (2009). Proyectos educativos innovadores, construcción y debate. México, CESU/UNAM.

Comenio, Juan Amós (2000). Didáctica Magna. México: Porrúa.

De Ketele, Jean Marie (2008). "Enfoque socio-histórico de las competencias en la Enseñanza". En: Profesorado. Revista de currículum y formación del profesorado, 12, 3. Recuperado el 15 de mayo de 2014 de: http://www.ugr.es/local/recfpro/rev123ART1.pdf

De la Torre, Miguel (2004). Del humanismo a la competitividad. México: UNAM/UANL.

Díaz-Barriga, Ángel (2011). "Competencias en educación. Corrientes de pensamiento e implicaciones para el currículo y el trabajo en el aula”, en Revista Iberoamericana de Educación Superior (RIES). Vol. II, núm. 5. México: UNAM-IISUE/Universia, [consulta: 17 de febrero 2013].2011). http://ries.universia.net/index.php/ries/article/view/126

Foucault, Michel (1986). Vigilar y castigar. Madrid: Siglo XXI.

Gadamer, Hans-Georg (1991-1992). Verdad y Método I y II. Salamanca: Sígueme.

Gimeno Sacristan, José (1990). La pedagogía por objetivos, obsesión por la eficiencia. Madrid: Morata.

Gonczi, A. (1996). Instrumentación de la educación basada en competencias. En: Competencia laboral y educación basada en normas de competencia. México: Limusa.

Lugo, Elisa (2008). Reformas educativas, su impacto en la innovación curricular y la formación docente. México. ANUIES/UAEM/Juan Pablos.

Martínez, López y Carpio (2013). "Análisis de la categoría competencia en los currícula de educación básica y media superior en México". Ponencia en el ler. Congreso internacional política, arte y narrativa en las configuraciones de lo filosófico-educativo. México: ATyFE. Memoria en $\mathrm{CD}$.

Mesnard, P. (1974). "La pedagogía de los jesuitas". En: Chateau. Los grandes pedagogos. México: Fondo de Cultura Económica.

Moreno Olivos, Tiburcio (2009). "Competencias en educación superior: un alto en el camino para revisar la ruta de viaje”. En: Perfiles Educativos. Vol. XXXI, núm. 124, 2009, pp. 69-92, Instituto de Investigaciones sobre la Universidad y la Educación. México: UNAM. 
OCDE (2005). The Definition and Selection of Key Competencies. Executive Summary (DeSeCo). www.oecd.org/edu/statistics/deseco

Palacios, Jesús (2007). La cuestión escolar, críticas y alternativas. México: Laia/ Coyoacán.

Pérez Arenas, David (2007). Filosofía, teoría e investigación en las maestrías en educación. Un campo sobredeterminado. México: Plaza y Valdez/UNAM ISSUE.

Pérez, Limón y García (2013). "Marginación, exclusión y/o desplazamiento de la formación relacionada con la filosofía, la epistemología y la teoría educativa en los posgrados en educación". En: Orozco y Pontón. Filosofía, teoría y campo de la educación 2002-2011. México, ANUIES/ COMIE.

Popkewitz, Thomas (2008). "Historia del currículum: una anotación breve en la historia". En: Profesorado. Revista de currículum y formación del profesorado, 12, 3, recuperado el 15 de mayo de 2014 de:

http://www.ugr.es/local/recfpro/rev113ed.pdf

Barry M. Franklin y Miguel A. Pereyra (coords.) (2003). Historia cultural y educación. Ensayos críticos sobre conocimiento y escolarización. Barcelona-México: Pomares.

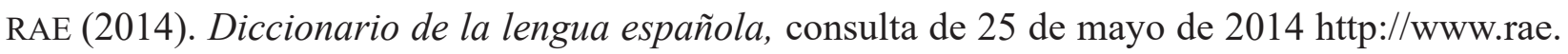
es/obras-academicas/diccionarios/diccionario-de-la-lengua-espanola

Navarro Leal (coord.) (2010). Educación Comparada: Perspectiva Latinoamericana. Ciudad Victoria: Sociedad Mexicana de Educación Comparada/Planeación Educación y Asesoría S.A. de C.V.

Rousseau, Jean-Jacques (2005). Emilio o la educación, Madrid: Alianza.

Santos Del Real, Annette Irene (2000). La educación secundaria: perspectivas de su demanda. México: Universidad Autónoma de Aguascalientes.

SEP (2011). Plan de estudios 2011. Educación Básica. México: SEP. /CONOCER (2008). Hacia un Sistema Nacional de Competencias de las Personas, que contribuya a la competitividad económica, al desarrollo educativo y al progreso social de todos los mexicanos.

ttp://www.conocer.gob.mx/pdfs/documentos/presentacion_conocer.pdf?view=wrap-per

Souto, Martha (1999). Grupos y dispositivos de formación. Buenos Aires: FFyL-UBA/Novedades Educativas.

Unión Europea (2010). "Education and Training 2010”, The Success of the Lisbon Strategy Hinges on Urgent Reforms. Recuperado de: http://europa.eu.int/comm/education/policies/2010/ et_2010_en.html 